\title{
The update of semantic memories in amnestic mild cognitive impairment
}

\author{
Alexandre de Mendonça', Sandra Cardoso', João Maroco², \\ Manuela Guerreiro' and Joana C. Carmo ${ }^{3 *}$ (ID \\ 'Faculdade de Medicina, Universidade de Lisboa, Portugal \\ ${ }^{2}$ Instituto Superior de Psicologia Aplicada, Lisboa, Portugal \\ ${ }^{3}$ Faculdade de Psicologia, Universidade de Lisboa, Portugal
}

It is still controversial to what extent neocortical consolidated memories are susceptible of change by processes of reconsolidation and transformation throughout experience, and whether the medial temporal lobes are necessary for this update of semantic consolidated memories, as they are for episodic remembering. We hypothesize that patients with amnestic mild cognitive impairment $(\mathrm{aMCl})$ who have deficits in episodic memory may also have difficulties in updating information on added new features of objects. Sixteen participants with aMCl and 20 healthy control participants performed a semantic word-to-picture task, in which they were asked to identify as belonging to a given semantic category NEW objects, that have incorporated novel features, as well as OLD items, semantically and visually SIMILAR items and UNRELATED items. Patients with aMCl made a greater percentage of errors than healthy controls. Participants globally made greater percentages of errors in difficult types of items, namely NEW and SIMILAR, as compared to easier ones, OLD and UNRELATED. Importantly, an item by diagnostic group interaction effect was observed, and post hoc analysis showed that patients with aMCl made a higher percentage of errors than controls in NEW items only. In conclusion, patients with aMCl had a particular difficulty in identifying the NEW items of the word-topicture task as compared to the control participants, supporting the concept of a flexible and dynamic conceptual knowledge system, involving the update of semantic memories and the integration of new attributes in a constant transformation process, which is impaired in these patients.

The distinction between two types of declarative memory, semantic and episodic, has a long tradition in cognitive neurosciences (Tulving, 1972), yet how these systems are organized in the brain is still a matter of discussion. On the one hand, episodic memory receives and stores information about dated events and temporal or spatial relations among these episodes. It is a contextual-dependent long-term representation of knowledge of past events. On the other hand, semantic memory is a mental thesaurus of organized knowledge about words and verbal symbols, concepts and their meanings and relations among them. It is a factual-based and context-free conceptual knowledge. In contrast to the episodic memory system, semantic memories are probably less susceptible to involuntary transformation and loss of information (Tulving, 1972, 1985).

* Correspondence should be addressed to Joana C. Carmo, Faculty of Psychology, University of Lisbon, Alameda da Universidade, 1649-0 I 3 Lisboa, Portugal (email: joanacostadocarmo@gmail.com). 
It is accepted that neural mechanisms, labelled consolidation processes, operate after the initial registration of information and contribute to the permanent storage of memory traces, as stated in the Standard Consolidation Theory of memory (Nadel \& Moscovitch, 1997). The retention and recovery of long-term memories are initially dependent upon the hippocampal formation and with the passage of time they eventually become independent of it (Squire \& Alvarez, 1995).

Following this line of research, McClelland, McNaughton, and O'Reilly (1995) advanced that the hippocampal function reflects a fast learning system which is complemented with a slow learning system by the neocortex. The neocortical system alone is optimized for the extraction of shared structures over the course of numerous repetitions and reinstatements, while the hippocampal system is optimized for pattern separation. Thus, for O'Reilly and Rudy (2000), episodic memory and this ability for generalization are complementary and work even in opposition.

In a further refinement of the latter ideas on memory consolidation, the Multiple Trace model (Nadel, \& Moscovitch, 1997) and its recent reformulation, the Memory Transformation Account (Winocur, \& Moscovitch, 2011; Winocur, Moscovitch, \& Bontempi, 2010) specified that (1) initially formed memories are episodic in nature and are dependent on the hippocampus for as long as they keep their episodic and contextual features; (2) with time and experience the hippocampus supports the development in the neocortex of a semanticized/schematic version of the original memory; and (3) dynamic nature of memory is emphasized since there is an interplay at retrieval between the two types of declarative memories (Winocur, \& Moscovitch, 2011; Winocur, Moscovitch, \& Bontempi, 2010).

Upon the work development on memory consolidation, another related concept has received a lot of interest, memory re-consolidation. Re-consolidation involves processes by which a fixed memory becomes once again labile, that is, a reactivated memory can suffer changes. These changes may have different directions, as memories can get weaker or erased (disruption processes), become stronger, or suffer alterations and updates which are dependent on novel (non-overlapping) experiences (Nadel, Hupbach, Gomez, \& Newman-Smith, 2012). It is claimed that a reactivation experience must involve reorganization in which new learning is incorporated (McKenzie \& Eichenbaum, 2011).

As a consequence of the advances in the understanding of memory consolidation and re-consolidation, the view of a stable and fixed memory as an end point of a consolidation process has been questioned (Nadel, Hupbach, Gomez, \& Newman-Smith, 2012). Some authors query the notion that consolidation operates in a way that new information is imposed on a tabula rasa, and instead believe that it must be interleaved within preexisting knowledge (McKenzie \& Eichenbaum, 2011). For Kroes and Fernández (2012), a dynamic system where memories are susceptible to change would be more efficient. However, they believe that stronger memories or abstract knowledge supported by cortical-cortical connections would be less susceptible of processes of re-consolidation (Kroes \& Fernández, 2012).

At this point, it is still an open question to what extent neocortical semanticized memories are susceptible of change by processes of reconsolidation or transformation (Kroes \& Fernández, 2012), in particular whether the medial temporal lobes are necessary for the update of memories or whether the neocortical memory system alone can do it. In accordance with the transformation account, the hippocampus would be necessary to the formation of schematic memories (Winocur, \& Moscovitch, 2011; Winocur, Moscovitch, \& Bontempi, 2010) and hence to the incorporation of new knowledge into existing 
schemas (update; McKenzie \& Eichenbaum, 2011; Nadel, Hupbach, Gomez, \& NewmanSmith, 2012).

Nowadays, a great deal of attention is paid to a group of patients who are at risk for developing dementia due to probable Alzheimer's disease in the ensuing years, a condition called amnestic Mild Cognitive Impairment (aMCI). These patients have normal general cognitive function and perform normal daily activities, yet they present abnormal memory function, below that expected for age and education (Peterson, 2004; Peterson et al., 1999). Episodic memory is particularly affected, reflecting atrophy of the hippocampus and related structures (Nunes et al., 2010).

Although semantic abilities are affected in dementia due to probable Alzheimer's disease (e.g., Adlam, Bozeat, Arnold, Watson, \& Hodges, 2006; Hodges et al., 1992), they have been found relatively preserved in aMCI, for instance in naming (Adlam et al., 2006; Albert, Moss, Tanzi, \& Jones, 2001; Balthazar et al., 2007), semantic knowledge association (Adlam et al., 2006) and similarities (Balthazar et al., 2007) tasks. Verbal fluency competencies have been also studied in aMCI patients. Some authors reported maintained performance in both semantic and letter fluency (Joubert et al., 2008), whereas others found semantic fluency to be impaired but not letter fluency (Adlam, et al., 2006; Clark et al., 2009; Vogel et al., 2005), this discrepancy being taken as evidence for an early degradation of the semantic system in aMCI (see Salmon, Heindel, \& Lange, 1999 for a similar discrepancy results in dementia due to probable Alzheimer's disease). In a longitudinal study with pre-clinical participants defined by biomarkers of amyloid burden, progression of the disease was associated with semantic fluency but not letter fluency (Papp et al., 2016). In another longitudinal study (Clark et al., 2009) recruiting participants initially with normal cognitive function who later developed AD, it was found instead that both letter and semantic fluency measures were associated with progression of the disease. Regarding language abilities, a study in patients at different stages of AD, confirmed by post-mortem brain examination, showed alterations in both syntactic and semantic features, increasing with later stages of the disease (Ahmed, Haigh, de Jager, \& Garrad, 2013).

Interestingly, using both with explicit (verbal fluency and sorting tasks in Hodges, et al., 1992) and implicit tasks (Laisney et al., 2011), patients with dementia due to probable Alzheimer's disease show impairments related to subordinate semantic levels or deterioration of distinctive semantic attributes, while shared, abstract features are relatively preserved. This pattern of results led the authors to assume a bottom-up breakdown in the structure of the semantic system, where high order abstract knowledge supported by shared features is preserved and unique and contextual features are most vulnerable (Hodges et al., 1992; Laisney et al., 2011). In the same line, Joubert et al. (2008) observed that patients with aMCI had greater difficulties with famous people and famous public events than common objects, interpreting this pattern as evidence for difficulties with conceptual entities having unique and distinctive attributes that are not shared by other member of a given category. Berisha et al. (2015) reported impairment in the use of unique words over time during the development of dementia due to probable Alzheimer's disease, which again might reflect a selective impact on the semantic system regarding specific and unique item attributes.

The differential pattern of relative preservation of semantic memory versus impairment of episodic memory, as well as the reported selective difficulties with conceptual entities having unique and distinctive semantic attributes, points out aMCI as an interesting condition to test the ability to update information and to add new features and attributes regarding old vocabulary of objects. Thus, in the present study, we tested a 
sample of patients with aMCI and control healthy individuals matched for age and schooling and gender in a semantic word-to-picture task. In each trial of this task, a name of an object was provided written on the top of the page and an array of six images below, that included OLD (e.g., old telephone or old mobile phone) and NEW items, for example (smartphone), as well as semantically and visually SIMILAR objects and also UNRELATED items. An adaptation of the Camel and Cactus test (CCT, Bozeat, Lambon Ralph, Patterson, Garrard \& Hodges, 2000), to control for semantic association abilities in the participants, was also applied.

Assuming that medial temporal lobe structures subserve and are a necessary component for both episodic memory processing and also for the update and integration of new features on pre-existing knowledge, we hypothesized that aMCI patients with deficits in episodic memory will also have selective difficulties in updating information on added new features of objects, showing deficits in identifying correctly the NEW objects in the word-to-picture task.

\section{Methods}

\section{Participants}

Sixteen participants with aMCI and 20 healthy control participants took part in the experiment. Patients were diagnosed with aMCI by a team of trained clinical professionals (neurologists and neuropsychologists) at a memory clinic, following a standard clinical, laboratory, neuroimaging and neuropsychological assessment. The controls were a convenience sample of patients' relatives and other volunteers in the community. The two samples were matched on age, schooling and gender. The study was approved by the Faculty's ethical committee, and all participants gave written informed consent prior to the experimental protocol. All participants had normal or corrected to normal vision.

\section{Inclusion criteria for all participants}

Participants were older than 55 years and had minimum of 4 years of formal education. They were all native speakers of European Portuguese.

\section{Inclusion criteria for the aMCl participants}

These criteria were adapted from Peterson et al. (1999):

1. presence of memory complains as reported by the participants or accompanying informants;

2. abnormal memory function as documented by the total recall score in list A, trials 1 to 5, in the California Verbal Learning Test (CVLT; Delis, Kramer, Kaplan \& Ober, 1987) lower than the cut-off of 1.5 standard deviation below the mean from the normative data;

3. normal general cognitive function documented by the Mini-Mental State Examination (MMSE) normalized for the Portuguese population (Folstein, Folstein \& McHugh, 1975);

4. no or minimal impairment in activities of daily living activities, that is, no more than one item from the Instrumental Activities of Daily Living (IADL) scale was abnormal (Lawton \& Brody, 1969). 
Inclusion criteria for the healthy controls

1. absence of memory complains;

2. normal memory function as documented by the total recall score in list A, trials $\mathbf{1}$ to 5 , in the CVLT above the cut-off of -1.5 standard deviation of the mean from the normative data;

3. normal general cognitive function documented by the MMSE;

4. no impairment in activities of daily living in IADL.

\section{Exclusion criteria for all participants}

1. diagnosis of dementia according to the Diagnostic and Statistical Manual of Mental Disorders - fourth Edition Revised (DSM-IV-R; American Psychiatric Association, 2000);

2. major depression according to DSM-IV-R;

3. history of neurological disorders, psychiatric conditions, uncontrolled medical illness or use of psychoactive medications able to interfere with cognition;

4. history of alcohol or drug abuse;

5. sensory deficits that could interfere with the assessment (Table 1).

\section{Material and procedure}

The neuropsychological assessment was carried out with the following instruments:

1. The MMSE (Folstein, Folstein \& McHugh, 1975) is a widely used brief instrument for the clinical evaluation of cognitive state in adults and the normative cut-off are adjusted to formal education level, in which there is indication of abnormal performance for scores below 22 for less than 11 years of schooling and below the score of 27 for individuals with more than 11 years of education. We applied the Portuguese version and normative data (Guerreiro et al., 1994);

2. The CVLT (Delis, Kramer, Kaplan, \& Ober, 1987) is a test of verbal learning that evaluates repetition learning, serial position effects, semantic organization, intrusion and proactive interference. There are two word lists (A and B) made up of 16 items from four different categories of 'shopping list items'. Participants are asked to

Table I. Participants' demographic and clinical characteristics

\begin{tabular}{lllr}
\hline & \multicolumn{1}{c}{ Controls } & \multicolumn{1}{c}{ aMCl } & p value \\
\hline$n$ & 20 & 16 & \\
Gender & 11 females: 9 males & 10 females: 6 males & .317 \\
Age (years) & $72.85(7.56)[6 I-86]$ & $74.50(7.70)[54-87]$ & .523 \\
Duration of memory complains (years) & & $4.19(4.37)[1-14]$ & \\
Schooling (years) & $11.55(5.37)[4-20]$ & $12.25(4.01)[4-16]$ & .667 \\
CVLT I to 5 (total) & $49.70(7.93)[38-65]$ & $30.81(5.4 I)[21-4 I]$ & $<.00 I^{*}$ \\
MMSE & $29.25(1.07)[27-30]$ & $27.25(2.08)[23-30]$ & $.00 I^{*}$ \\
\hline
\end{tabular}

Mean, standard deviation and interval range are provided for Age, Schooling, performance on the California Verbal Learning test (CVLT) for Total immediate trials and Mini-Mental State Examination (MMSE) test scores. Student's $t$-tests were calculated for all variables, except for Gender where the chisquare test was used. $p$ values $<.05$ are identified with *. 
retrieve the list of items immediately in five repetition trials (list A) and one repetition for list $\mathrm{B}$; list $\mathrm{A}$ is recalled again after a long delay. We used the total number of correctly recalled words of list A, trials 1 to 5, (Immediate Total Recall) to distinguish between the two samples (see Silva et al., 2012, this score was the best predictor of progression of MCI to $\mathrm{AD}$ for a cut-off of $1.5 S D$ below the mean of normative data). The Portuguese version of the CVLT was used (Ribeiro et al., 2007).

3. The IADL Scale is an instrument that evaluates in eight questions the functionality on instrumental activities of daily life and self-care (Lawton and Brody (1969). The Portuguese version of this test was used (Pantoni et al., 2005).

Two experimental tasks were applied to each participant in a fixed order and in a paper and pencil format.

1. The word-to-picture task was designed to evaluate the semantic update of items in an adaptation from Lambon-Raph et al. (2010). In each trial, subjects were presented with an assorted array of six images and were asked to indicate which images corresponded to a target name on the top of the page (see Figure 1). The target items could be OLD (e.g., a mobile phone) and NEW items (e.g., a smartphone). In each trial, besides the target items that could vary from 2 to 4 , there were also semantically and visually SIMILAR items (e.g., a calculator) and also UNRELATED items (e.g., kitchen utensils). Both SIMILAR and UNRELATED items could vary from 1 to 3 and could appear repeated (e.g., 2 calculators) in some trials (five in total). Objects that had suffered alterations in one or more features (e.g., touchscreen in smartphones instead of keyboard) were considered NEW items. A pre-test was run with initial 18 different arrays of objects. Ten participants (age: $M=70.9 ; S D=6.0$; range [65-81]; schooling (in years): $M=11.5 ; S D=4.8$; range $=[4-16]$ ) participated in the pretest, and arrays that had less than $60 \%$ correct responses on target objects were excluded. The final test comprises 12 trials, in which objects that had suffered alterations in one or more features (e.g., touchscreen in smartphones instead of keyboard) were considered NEW items (please see the Supporting Information for a complete of list of objects used in the 12 trials). The trials were randomized as well as the position of the items.

2. The Camel and Cactus test (CCT; Bozeat et al., 2000) a cultural adaptation of the test from our laboratory (unpublished material) was used (Figure 2). In this version, all 64 trials were kept but the figures were now colour photographs of the same items. This is a test of semantic association where in each trial four alternatives of the same category (e.g., fruits) are given to the participant and they have to choose which of the four items is more associated with a target image on the top of the page (e.g., bottle of wine associated with grapes). The experimenter registers the answer of the participants in the tasks applied.

\section{Statistical analysis}

Comparison of the demographic variables between the two groups was performed with the Student $t$-test for numerical variables: Age, Schooling, CVLT Total immediate score and MMSE score. For the categorical variable Gender, the chi-square test was used to compare gender distributions between groups.

For the word-to-picture task, the percentage of errors was used since the total possible amount of each error type was different for each category. A repeated measures ANOVA 
Identify all mobile phones:

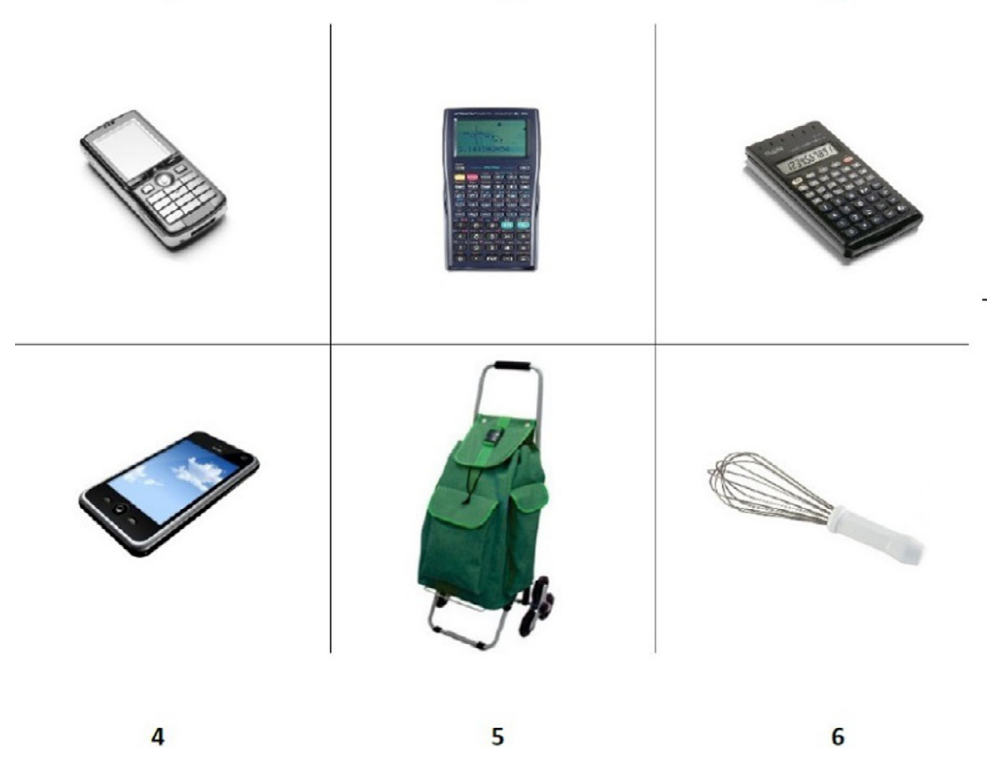

Figure I. Example of a trial in the word-to-picture task of semantic update. An array of six pictures is presented in each trial. On top of the page, the question is presented and referenced to the target object.

was run for the percentage of errors with group (aMCIs, Controls) as between subjects variable and item type (OLD, NEW, SIMILAR, UNRELATED) as within subjects factor. To identify significant differences among the four item types, within-subjects contrasts analysis was performed with Bonferroni's correction for six comparisons. To specify item by group interaction effects, Student's $t$-tests were run for each error type (OLD, NEW, SIMILAR, UNRELATED), variances were considered not equal after Levene's test, and the correction of Bonferroni for four comparisons was applied.

Comparison of the percentages of errors in the Camel and Cactus test in patients with aMCI and controls was done with the Student's $t$-test.

For all the analysis, IBM SPSS Statistics 23 for Windows (SPSS Inc.) was used. Values of $p<.05$ were considered statistically significant. Effect sizes were interpreted following Cohen (1988) both for Cohen's $d$ and $\eta_{\mathrm{p}}^{2}$.

\section{Results}

\section{Word-to-picture task}

In the word-to-picture task (Figure 3), a main effect of group was found significant ( $F$ $(1,34)=19.721, p<.001, \eta_{\mathrm{p}}^{2}=0.367$, large effect size), patients with aMCI making a greater percentage of errors than healthy controls (see Table 2). There was also a significant difference in the item type $\left(F(3,32)=32.574, p<.001, \eta_{\mathrm{p}}^{2}=0.489\right.$, large effect size). Interestingly, a type of item by group interaction effect $(F(3,32)=5.744$, $p=.001, \eta_{\mathrm{p}}^{2}=0.145$, large effect size) was observed. To specify this interaction effect, subsequent Student's $t$-tests with Bonferroni's correction were performed to compare the percentages of errors between aMCI patients and controls for each item type (OLD, NEW, 


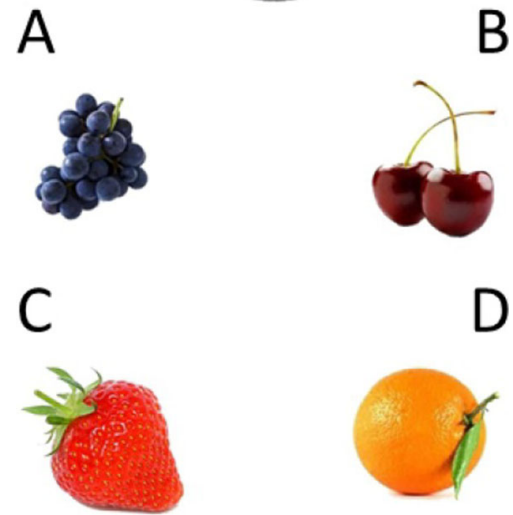

Figure 2. Example of a trial of the Camel and Cactus test (CCT) adaptation. In each trial, the target item is presented on top of the page and below four alternative responses are shown.

SIMILAR, UNRELATED). A statistically significant difference was found where patients with aMCI showed a higher percentage of errors $(S k=0.61, K u=-0.37)$ than controls $(S k=1.81, K u=3.54)$ for the NEW items $(t(34)=3.535, p=.001$, Cohen's $d=1.16$, large effect size), no differences being observed for OLD, SIMILAR and UNRELATED items.

In a secondary analysis to identify the significant differences among the item types, using the subjects contrasts, NEW items and SIMILAR items had similar percentages of errors $(F(1,34)=4.032, p>.05)$. NEW items had higher percentage of errors than both OLD items $(F(1,34)=51.260, p<.001)$ and UNRELATED items $(F(1,34)=59.434$, $p<.001)$, and in an analogous way, SIMILAR items showed higher percentage of errors than both OLD items $(F(1,34)=30.677, p<.001)$ and UNRELATED items ( $F$ $(1,34)=38.384, p<.001)$. Finally, participants did lower and not statistically different percentages of errors in OLD items and UNRELATED items $(F(1,34)=3.969, p>.05)$.

\section{Camel and Cactus test}

For the Camel and Cactus Test (see Table 2), no significant differences in the percentage of errors were observed $(t(34)=1.84, p>.05$, Cohen's $d=0.61$ (moderate effect) between the patients with aMCI $(S k=-0.23, K u=-1.58)$ and controls $(S k=0.71$, $K u=-0.94)$.

\section{Discussion}

The main finding of the present study is that patients with aMCI, who have deficits in episodic memory and a relative preservation of the lexical-semantic components of memory, show difficulties to update semantic information, that is to accomplish the vocabulary update of objects that have changed features over time with the addition of new attributes. 


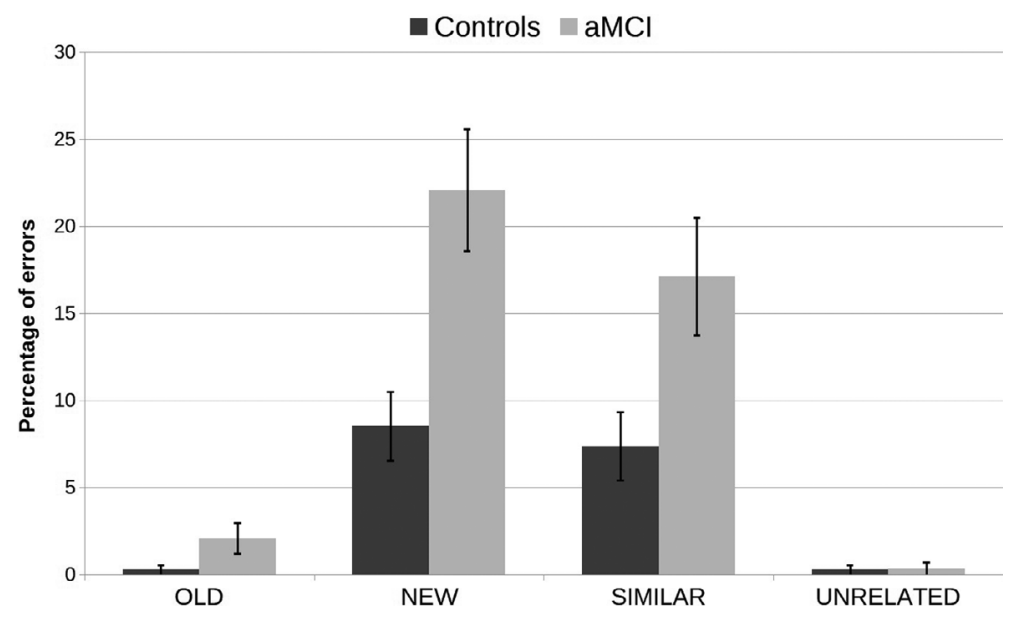

Figure 3. The average percentage of errors is depicted for the different item types (OLD, NEW, SIMILAR, UNRELATED) in the aMCl and control groups. Error bars are the standard error of the mean. Globally, patients with aMCl made a greater percentage of errors than controls. Interestingly, an interaction effect was found, showing that patients with aMCl had particular difficulty in the NEW items of the word-to-picture task (see the main text for details on the statistical analysis).

Table 2. Results for the Camel and Cactus test (adaptation; CCT) and for the word-to-picture test for both the control and aMCl samples

\begin{tabular}{lccc}
\hline Tests & Controls & $\mathrm{aMCl}$ & Total \\
\hline CCT & $12.74(9.32)$ & $18.36(8.8 \mathrm{I})$ & $\mathrm{I} 5.23(9.4 \mathrm{I})$ \\
Word-to-picture & & & \\
Old items & $0.28(1.24)$ & $2.08(3.44)$ & $\mathrm{I} .08(2.59)$ \\
New items & $8.52(8.84)$ & $22.06(14.00)$ & $14.54(13.15)$ \\
Similar items & $7.37(8.77)$ & $17.1 \mathrm{I}(13.52)$ & $11.69(12.0 \mathrm{I})$ \\
Unrelated items & $0.28(1.24)$ & $0.35(1.39)$ & $0.31(1.29)$ \\
Total & $4.1 \mathrm{I}(7.59)$ & $19.39(13.52)$ & \\
\hline
\end{tabular}

Mean (standard deviation) are provided for percentage of errors for both tasks.

The experimental word-to-picture task was designed to evaluate the flexibility of semantic categories content by integration of novel information. It is an adaptation of the match-to-sample task used by Lambon-Raph et al. (2010), focusing rather on selected objects that have changed features over time. The critical items from the presented array of objects are the NEW items. This type of item should be correctly identified as belonging to the given category name if the semantic content of the category has been updated to incorporate these new objects. The participants are presented, in each trial, with an array of six different pictures and are asked to identify all items that are part of a given name category (e.g., mobile phones). Participants would have to correctly choose NEW items that are versions of old objects but with added novel features. The array also comprises OLD items, UNRELATED items and semantically and visually SIMILAR objects.

The main result was that patients with aMCI had a particular difficulty in identifying the NEW items of the word-to-picture task as compared to the control participants. The 
difficulties in identifying NEW items reveal that objects that are novel seem not being processed as part of the naming category and could be processed as a rather new semantic concept. Because the patients with aMCI in this study evidence episodic memory deficits and are therefore likely to have involvement of the medial temporal lobe, their difficulty with identifying NEW items may suggest that the middle temporal lobe may be necessary for the update of novel, non-overlapping features of objects and that dysfunction of the hippocampal formation system and related structures may hinder the update of daily objects concepts with their added new features. In other words, the slow neocortical system may be necessary but not sufficient for the incorporation of new conceptual knowledge into previously stored schemas (McKenzie \& Eichenbaum, 2011; Nadel, Hupbach, Gomez, \& Newman-Smith, 2012). Kroes and Fernández (2012) emphasize that abstract, cortical memories need keeping a flexible nature, yet they state that these memories would be less susceptible of change through hippocampaldependent consolidation and transformation processes (Kroes \& Fernández, 2012). The present observation that patients with episodic memory impairments also show difficulties with the integration of new information into old semanticized memories might indicate that transformation and flexible updating of abstract conceptual knowledge might be recruiting the same medial temporal lobes for a dynamic efficient memory function. From this point of view, the present findings support and also extend the Memory Transformation Account theory (Winocur, \& Moscovitch, 2011; Winocur, Moscovitch, \& Bontempi, 2010), in the sense that the hippocampus is involved, not only in the initial formation of schematic memories, but also in the additional transformations of stored schemas backed up by subject's experience, in a continuous renovation process.

Interestingly, the selective difficulties that patients with aMCIs showed in identifying NEW objects were observed despite a general preservation of the semantic neocortical system, as revealed by a normal performance it the Camel and Cactus test of semantic association. This could just reflect a high degree of difficulty of the experimental word-topicture task, comprising visually and semantically similar items, with a variable number of correct responses across trials. In other words, the word-to-picture task might be more sensitive than the Camel and Cactus test to identify semantic impairments in patients with aMCI. Another possibility is that the neocortical semantic system alone might be relatively preserved, but the complex connectivity between the hippocampal and the neocortical system might be hindered. This would be consistent with reports emphasizing the importance of functional connectivity between the hippocampus and neocortical areas, for instance Takashima et al. (2009) described a functional shift in the connectivity networks during memory consolidation, namely decreased connectivity between hippocampal structures and neocortical areas, together with enhanced cortico-cortical connectivity.

As expected, participants, both aMCI patients and healthy controls, made a larger percentage of errors in more difficult types of items of word-to-picture task, namely NEW and SIMILAR items, as compared to easier ones, OLD and UNRELATED items, but it is noteworthy that patients with aMCI globally made a greater percentage of errors than controls. This result presumably reflects mild widespread deficiencies in cognitive domains essential to accomplish a complex task, namely the ability to maintain attention for long periods or decision-making capabilities (see for instance, Coelho et al, 2017, 2019). Accordingly, patients with aMCI had lower, albeit normal, scores on a general evaluation of the cognitive state with the MMSE. In any case, the absence of differences between patients with aMCI and controls on the Camel and Cactus test would ensure that 
major visual perceptive deficits, language difficulties or general semantic capabilities might have impacted little on the main results of the study. These results on the Camel and Cactus test are in accordance with reports of preserved semantic system components in patients with aMCI (Balthazar et al., 2007) and maintained performance in a test of semantic association (Adlam et al., 2006).

The use of everyday technology (ET) in patients with in mild cognitive impairment has been addressed in previous studies (e.g., Bartels et al., 2019; Nygård, 2008). Patients with aMCI have difficulties with knowledge of objects regarding their action and function (Adlam et al., 2006). The perceived ability to use ET declines over time, accompanying the functional deterioration in patients with aMCI (Hedman, Kottorp, Almkvist \& Nygard, 2018). Many of the objects presented to the participants in our study represent technology of general use nowadays, so that the deficits that patients with aMCI showed regarding the integration of these novel objects into the semantic system could affect the use of these objects, and increase the perceived difficulty of using these objects. Thus, training the semantic update of objects that have suffered alterations in recent years might well be a reasonable cognitive rehabilitation strategy in patients with aMCI.

Some limitations of the current work must be mentioned. In first place, the sample size was limited. In second place, the semantic abilities were assessed using visual tasks, so that the results might not generalizable to other semantic systems. In third place, no direct measurements of the medial temporal lobe structures were available. Finally, it would be interesting to incorporate a measure of access and exposure to technology in future studies, to understand better the relationship among the conceptual semantic knowledge of new objects, the perceived ability and the actual skill to manipulate them. We recognize that since the sample size is limited, and hippocampal volume measurements were not performed, the results of this study must be considered preliminary, fostering further investigation along this innovative line of research.

In conclusion, the present work supports the concept of a flexible and dynamic conceptual knowledge system, involving the update of semantic memories and the integration of new attributes in a constant transformation process, which is impaired in patients with aMCI.

\section{Acknowledgements}

The present work was financed by national funding through FCT - Fundação para a Ciência e a Tecnologia, I. P., on the scope of Norma Transitória DL57/2016/CP1439/CT02. The funders had no role in study design, data collection and analysis, or preparation of the manuscript. We sincerely thank all the participants that took part in this study as well as the Memo Clinica for all the support in the recruitment and testing.

\section{Conflicts of interest}

All authors declare no conflict of interest.

\section{Author contribution}

Joana C. Carmo, Ph.D. (Conceptualization; Data curation; Formal analysis; Investigation; Methodology; Writing - original draft) Alexandre de Mendonça (Investigation; 
Methodology; Supervision; Writing - review \& editing) Sandra Cardoso (Investigation; Methodology; Resources) João Maroco (Formal analysis) Manuela Guerreiro (Methodology; Resources; Supervision; Writing - review \& editing).

\section{Data availability statement}

The data that support the findings of this study are available on request from the corresponding author. The data are not publicly available due to privacy or ethical restrictions.

\section{References}

Adlam, A.-L. R., Bozeat, S., Arnold, A., Watson, P., \& Hodges, J. R. (2006). Semantic knowledge in mild cognitive impairment and mild Alzheimer's disease. Cortex, 42, 675-684. https://doi.org/10. 1016/S0010-9452(08)70404-0

Ahmed, S., Haigh, A.-M. F., de Jager, C. A., \& Garrad, P. (2013). Connected speech as a marker of disease progression in autopsy-proven Alzheimer's disease. Brain, 136, 3727-3737. https://doi. org/10.1093/brain/awt269

Albert, M. S., Moss, M. B., Tanzi, R., \& Jones, K. (2001). Preclinical prediction of AD using neuropsychological tests. Journal of the International Neuropsychological Society, 7, 631639. https://doi.org/10.1017/S1355617701755105

American Psychiatric Association. (2000). Diagnostic and statistical manual of mental disorders (4th ed.). Washington, DC: Author.

Balthazar, M. L. F., Martinelli, J. E., Cendes, F., \& Damasceno, B. P. (2007). Lexical semantic memory in amnestic mild cognitive impairment and mild Alzheimer's disease. Arquives of Neuropsychiatry, 65, 619-622. https://doi.org/10.1590/S0004-282X2007000400014

Bartels, S. L., Assander, S., Patomella, A.-H., Jamnada-Khoda, J., \& Malinowsky, C. (2019). Do you observe what I perceive? The relationship between two perspectives on the ability of people with cognitive impairments to use everyday technology. Aging \& Mental Health. https://doi. org/10.1080/13607863.2019.1609902

Berisha, V., Wang, S., LaCross, A., \& Liss, J. (2015). Tracking discourse complexity preceding Alzheimer's disease diagnosis: A case study comparing the press Conferences of Presidents Ronald Reagan and George Herbert Walker Bush. Journal of Alzheimer's Disease, 45(3), 959963. https://doi.org/10.3233/jad-142763

Bozeat, S., Lambon Ralph, M. A., Patterson, K., Garrard, P., \& Hodges, J. R. (2000). Non-verbal semantic impairment in semantic dementia. Neuropsychologia, 38, 1207-1215. https://doi. org/10.1016/S0028-3932(00)00034-8

Clark, L. J., Gatz, M., Zheng, L., Chen, Y.-L., \& McCleary, C., Mack, W. J. (2009). Longitudinal verbal fluency in normal aging, preclinical, and prevalent Alzheimer's disease. American Journal of Alzheimer's Disease \& Other Dementiasr, 24(6), 461-468. https://doi.org/10.1177/ 1533317509345154

Coelho, S., Guerreiro, M., Chester, C., Silva, D., Maroco, J., Paglieri, F., \& de Mendonça, A. (2017). Delay discounting in mild cognitive impairment. Journal of Clinical and Experimental Neuropsychology, 39, 336-346. https://doi.org/10.1080/13803395.2016.1226269

Coelho, S., Guerreiro, M., Chester, C., Silva, D., Maroco, J., Paglieri, F., \& de Mendonça, A. (2019). Mental time travel in mild cognitive impairment. Journal of Clinical and Experimental Neuropsychology, 41, 845-855. https://doi.org/10.1080/13803395.2019.1632269

Cohen, J. (1988). Statistical power analysis for the behavioral sciences. New York, NY: Routledge Academic.

Delis, D. C., Kramer, J. H., Kaplan, E., \& Ober, B. A. (1987). The California verbal learning test: Research edition adult version. San Antonio, TX: The Psychological Corporation. 
Folstein, M. F., Folstein, S. E., \& McHugh, P. R. (1975). 'Mini-Mental State': A practical method for grading the cognitive state of patients for clinician. Journal of Psychiatry Research, 12, 189198. https://doi.org/10.1016/0022-3956(75)90026-6

Guerreiro, M., Silva, A. P., Botelho, M. A., Leitão, O., Castro Caldas, A., \& Garcia, C. (1994). Adaptação à população portuguesa na tradução do "MiniMental State Examination" (MMSE). Revista Portuguesa de Neurologia., 1, 9.

Hedman, A., Kottorp, A., Almkvist, O., \& Nygård, L. (2018). Challenge levels of everyday technologies as perceived over five years by older adults with mild cognitive impairment. International Psychogeriatrics, 30(10), 1447-1454. https://doi.org/10.1017/s104161021 8000285

Hodges, J. R., Salmon, D. P., \& Butters, N. (1992). Semantic memory impairment in Alzheimer's disease: Failure of access or degraded knowledge? Neuropsychologia, 30(4), 301-314. https:// doi.org/10.1016/0028-3932(92)90104-t

Joubert, S., Felician, O., Barbeau, E., Didic, M., Poncet, M., \& Ceccaldi, M. (2008). Patterns of semantic memory impairment in mild cognitive impairment. Behavioural Neurology, 19, 3540. https://doi.org/10.1155/2008/859657

Kroes, M. C. W., \& Fernández, G. (2012). Dynamic neural systems enable adaptive, flexible memories. Neuroscience and Biobehavioral Reviews, 36, 1646-1666. https://doi.org/10. 1016/j.neubiorev.2012.02.014

Laisney, M., Giffard, B., Belliard, S., de la Sayette, V., Desgranges, B., \& Eustache, F. (2011). When the zebra loses its stripes: Semantic priming in early Alzheimer's Disease and Semantic Dementia. Cortex, 47, 35-46. https://doi.org/10.1016/j.cortex.2009.11.001

Lambon-Raph, M. A., Sage, K., Jones, R. W., \& Mayberry, E. J. (2010). Coherent concepts are computed in the anterior temporal lobes. Proceedings of the National Academy of Science, 107, 2717-2722. https://doi.org/10.1073/pnas.0907307107

Lawton, M. P., \& Brody, E. M. (1969). Assessment of older people: Self-maintaining and instrumental activities of daily live. The Gerontologist, 9, 179-186. https://doi.org/10.1093/geront/9.3_Part_ 1.179

McClelland, J. L., McNaughton, B. L., \& O'Reilly, R. C. (1995). Why there are complementary learning systems in the hippocampus and neocortex: Insights from the successes and failures of connectionist models of learning and memory. Psychological Review, 102, 419-457. https:// doi.org/10.1037/0033-295X.102.3.419

McKenzie, S., \& Eichenbaum, H. (2011). Consolidation and reconsolidation: Two lives of memories? Neuron, 71, 224-233. https://doi.org/10.1016/j.neuron.2011.06.037

Nadel, L., Hupbach, A., Gomez, R., \& Newman-Smith, K. (2012). Memory formation, consolidation and transformation. Neuroscience and Biobehavioral Reviews, 36, 1640-1645. https://doi. org/10.1016/j.neubiorev.2012.03.001

Nadel, L., \& Moscovitch, M. (1997). Memory consolidation, retrograde amnesia and the hippocampal complex. Current Opinion in Neurobiology, 7, 217-227. https://doi.org/10. 1016/S0959-4388(97)80010-4

Nunes, T., Fragata, I., Ribeiro, F., Palma, T., Maroco, J., Cannas, J., ... de Mendonça, A. (2010). The outcome of elderly patients with cognitive complaints but normal neuropsychological tests. Journal of Alzheimer's Disease, 19(1), 137-145. https://doi.org/10.3233/JAD-2010-1210

Nygård, L. (2008). The meaning of everyday technology as experienced by people with dementia who live alone. Dementia, 7, 481-502. https://doi.org/10.1177/1471301208096631

O'Reilly, R. C., \& Rudy, J. W. (2000). Computational principles of learning in the neocortex and hippocampus. Hippocampus, 10, 389-397. https://doi.org/10.1002/1098-1063(2000)10: 4<389:AID-HIPO5>3.0.CO;2-P

Pantoni, L., Basile, A., Pracucci, J., Asplund, K., Bogousslavsky, J., Chabriat, H., . . Inzitari, D. (2005). Impact of age related cerebral white matter changes on the transition to disability - The Ladis study: Rationale, design and methodology. Neuroepidemiology, 24, 51-62. https://doi.org/10. 1159/000081050 
Papp, K., Mormino, E. C., Amariglio, R. E., Munro, C., Dagley, A., Schultz, A. P., Johnson, K. A., Sperling, R. A., \& Renz, D. M. (2016). Biomarker validation of a decline in semantic processing in preclinical Alzheimer's disease. Neuropsychology, 30, 624-630. https://doi.org/10.1037/ne u0000246

Peterson, R. C. (2004). Mild cognitive impairment as a diagnostic entity. Journal of Internal Medicine, 256, 183-194. https://doi.org/10.1111/j.1365-2796.2004.01388.x

Peterson, R. C., Smith, G. E., Waring, S. C., Ivnik, R. J., Tangalos, E. G., \& Kokmen, E. (1999). Mild cognitive impairment: Clinical characterization and outcome. Archives of Neurology, 56, 303308. https://doi.org/10.1001/archneur.56.3.303

Ribeiro, F., Guerreiro, M., \& de Mendonça, A. (2007). Verbal learning and memory deficits in mild cognitive impairment. Journal of Clinical and Experimental Neuropsychology, 29, 187-197. https://doi.org/10.1080/13803390600629775

Salmon, D. P., Heindel, W. C., \& Lange, K. L. (1999). Differential decline in word generation from phonemic and semantic categories during the course of Alzheimer's disease: Implications for the integrity of semantic memory. Journal of the International Neuropsychological Society, 5(7), 692-703. https://doi.org/10.1017/s1355617799577126

Silva, D., Guerreiro, M., Maroco, J., Santana, I., Rodrigues, A., Marques, J. B., \& Mendonça, A. (2012). Comparison of four verbal memory tests for the diagnosis and predictive value of mild cognitive impairment. Dementia and Geriatric Cognitive Disorders Extra, 2, 120-131. https://doi.org/ $10.1159 / 000336224$

Squire, L. R., \& Alvarez, P. (1995). Retrograde amnesia and memory consolidation: A neurobiological perspective. Current Opinion in Neurobiology, 5, 169-177. https://doi.org/10.1016/09594388(95)80023-9

Takashima, A., Nieuwenhuis, I. L. C., Jense, O., Talamini, L. M., Rijpkema, M., \& Fernandez, G. (2009). Shift from hippocampal to neocortical centered retrieval network with consolidation. Journal of Neuroscience, 29, 10087-10093. https://doi.org/10.1523/JNEUROSCI.0799-09. 2009

Tulving, E. (1972). Episodic and semantic memory. In E. Tulving \& W. Donaldson (Eds.), Organization of memory. New York, NY: Academic Press.

Tulving, E. (1985). Memory and consciousness. Canadian Psychology, 26(1), 1-12. https://doi.org/ $10.1037 / \mathrm{h} 0080017$

Vogel, A., Gade, A., Stokholm, J., \& Waldemar G. (2005). Semantic memory impairment in the earliest phases of Alzheimer's disease. Dementia and Geriatric Cognitive Disorders, 19(2-3), 75-81. https://doi.org/10.1159/000082352

Winocur, G., \& Moscovitch, M. (2011). Memory transformation and system consolidation. Journal of International Neuropsychological Society, 17, 766-780. https://doi.org/10.1017/ S1355617711000683

Winocur, G., Moscovitch, M., \& Bontempi, B. (2010). Memory formation and long-term retention in humans and animals: Convergence towards a transformation account of hippocampalneocortical interactions. Neuropsychologia, 48, 2339-2356. https://doi.org/10.1016/j.ne uropsychologia.2010.04.016

Received I 8 July 2019; revised version received I April 2020

\section{Supporting Information}

The following supporting information may be found in the online edition of the article:

Table S1. List of items used for each category: NEW, OLD, Visually and semantic SIMILAR and UNRELATED items. 